\title{
Neutron Multiple Number as a Factor Ruling Both the Abundance and Some Material Properties of Elements
}

\author{
Yoshiharu Mae ${ }^{1}$ \\ ${ }^{1}$ Maetech, Saitama, Japan \\ Correspondence: Yoshiharu Mae, Maetech, 685-8, Mimuro, Midori-ward, Saitama-city, 336-0911, Japan. \\ E-mail: yoshimae@hotmail.com
}

Received: June 14, 2017

Accepted: June 27, 2017

Online Published: June 30, 2017

doi:10.5539/jmsr.v6n3p37

URL: https://doi.org/10.5539/jmsr.v6n3p37

\begin{abstract}
The abundance of elements in the universe was plotted on the TC-YM diagram. The most abundant elements show the unique pattern drawing a quadrant. Next, the neutron multiple number, the number of neutron per proton in the nucleus, was introduced. The neutron multiple numbers of elements show the same pattern as the abundance of elements on the diagram. As a result, the abundance of elements shows a good correlation with neutron multiple numbers of elements. With increasing neutron multiple number, the abundance decreases. Besides, the neutron multiple number relates to the materials properties such as the Young's modulus, thermal conductivity and melting temperature of elements.
\end{abstract}

Keywords: abundance, neutron, proton, nucleus, Young's modulus, thermal conductivity, melting point

\section{Introduction}

Elements in the periodic table are almost metallic or semi-metallic elements. The periodic table is a great expression of these elements, but it is difficult to apply it to the specific metallurgical events. In general, main characteristics of the metallic elements are crystal structures and the existence of free electrons. Among many physical properties, the Young's modulus was selected as an index representing the strength of lattice and the thermal conductivity as an index representing the characteristics of free electrons. Consequently, a diagram with the thermal conductivity on the abscissa and the Young's modulus on the ordinate was made, and each element was plotted on the diagram. This diagram will be referred to as the "thermal conductivity-Young's modulus" (TC-YM) diagram.

The TC-YM diagram shows clear patterns with respect to the crystal structures of elements, and other elemental properties, such as atomic radius, melting point, thermal expansion, boiling point, heat of fusion, vapor pressure, heat capacity, electronegativity, and ionization energy.

\section{Abundance of Elements in the Universe}

The abundance of elements in the universe is often shown in books (Ono, 2008; Oda, 1987). Figure 1 shows the abundance of elements in the universe on the TC-YM diagram.

The abundance is the existing ratio when the content of $\mathrm{Si}$ is normalized to 1,000,000. The figures in the legend show the range of the abundance in the logarithmic scale. The elements of the largest abundance $\mathrm{Mn}, \mathrm{Cr}, \mathrm{Fe}, \mathrm{Ni}$, $\mathrm{Co}, \mathrm{Si}, \mathrm{Al}, \mathrm{Zn}, \mathrm{Mg}, \mathrm{Ca}, \mathrm{Na}$ and $\mathrm{K}$ unexpectedly form a quadrant on the diagram, and the elements with smaller abundance are distributed inside or outside the quadrant.

This pattern is quite a new one, and is different from any other patterns observed in other properties. The abundance is not a physical property of the elements. It is a result of the nuclear reactions during the origin of the universe. Why such a factor does have a unique pattern on the TC-YM diagram?

\section{Introduction of the Neutron Multiple Number}

The atomic mass is a sum of the number of protons and neutrons (Kumano, 2015). When the difference between atomic mass and atomic number is divided by atomic number, the quotient means the number of neutron per proton in nucleus. It will be referred to as the "neutron multiple number" hereafter.

Figure 2 shows the distribution of this "neutron multiple number" of elements on the TC-YM diagram. 


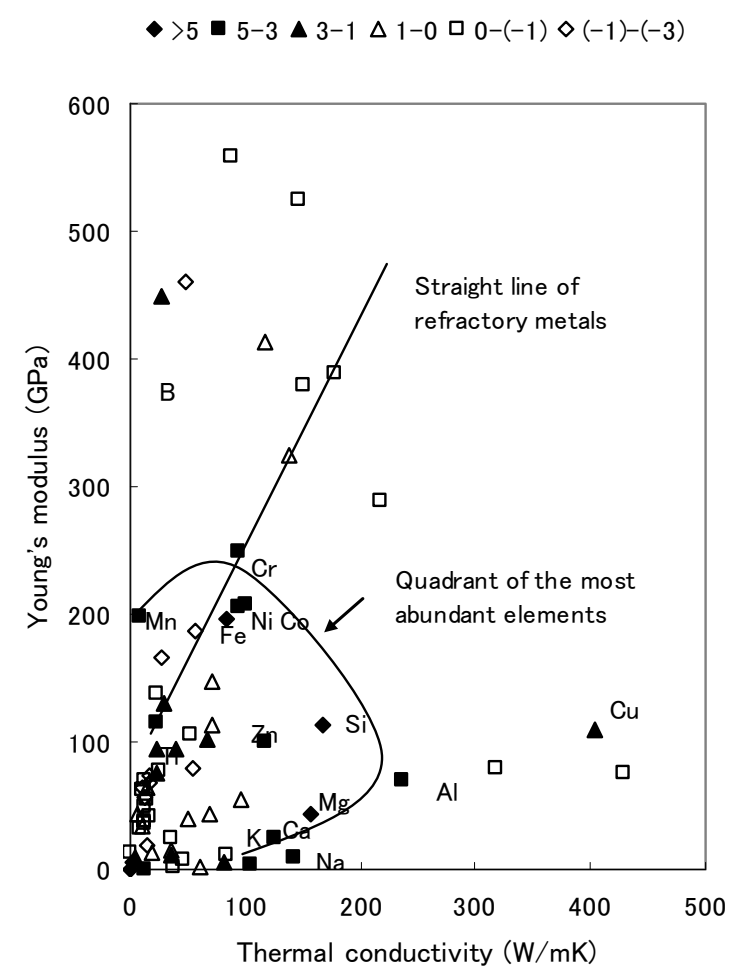

Figure 1. Distribution of the abundance (log abundance) of elements in the universe on the diagram
• 1-1.1 $\Delta 1.1-1.2 \Delta 1.2-1.3 \square 1.3-1.4 \diamond 1.4-1.5 \times 1.5-1.6$

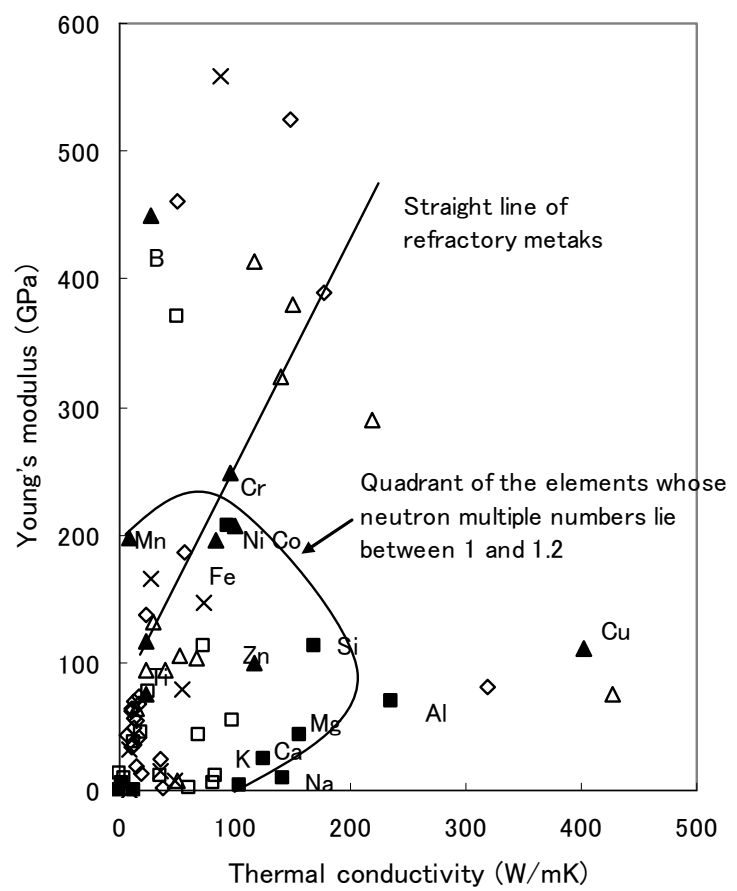

Figure 2. Distribution of the neutron multiple number of elements on the diagram

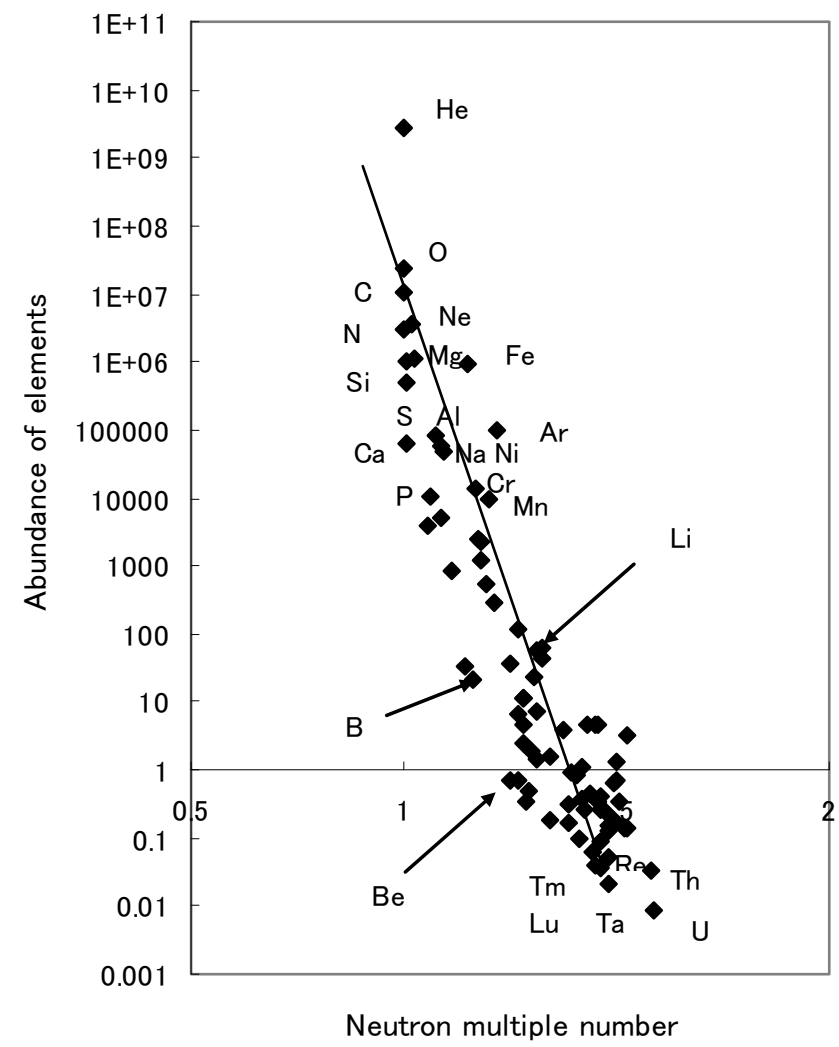

Figure 3. Correlation between the abundance of elements in the universe and the neutron multiple number of elements 
The figures in the legend show the range of the neutron multiple number. Surprisingly, the neutron multiple number of elements shows a pattern on the diagram. The elements with the smallest neutron multiple number Mn, $\mathrm{Cr}, \mathrm{Fe}, \mathrm{Ni}, \mathrm{Co}, \mathrm{Si}, \mathrm{Al}, \mathrm{Zn}, \mathrm{Mg}, \mathrm{Ca}, \mathrm{Na}$ and $\mathrm{K}$ form a quadrant on the diagram. Elements of large neutron multiple number are distributed inside or outside the quadrant. It is quite a new pattern and absolutely different from any other patterns obtained so far. It is surprising that the character of nucleus such as the neutron multiple number is related to the physical properties of materials like Young's modulus and thermal conductivity. Moreover, it is the same pattern as the abundance of elements in the universe shown in Figure 1. Therefore, a good correlation between them is expected. Figure 3 shows the correlation between the abundance of elements in the universe and the neutron multiple number of elements.

Good correlation is seen. The "neutron excess" is defined as the difference between the number of neutrons and the number of protons (Aller, 1961). But this neutron multiple number seems not to have been quoted yet, and Figure 3 seems not to have been shown before. As the neutron multiple number increases, the abundance of the element decreases.

The elements are created in the universe by many sorts of process (Aller, 1961; Aoki, 2004). Figure 3 shows that the probability of the creating reaction of each element is dependent on the fact how many neutrons it needs for its protons. The element which needs more neutrons for its protons is difficult to be created.

Figure 4 is the Oddo-Harkins plot with respect to the abundance of elements. With increasing atomic number, the abundance of elements decreases rapidly.

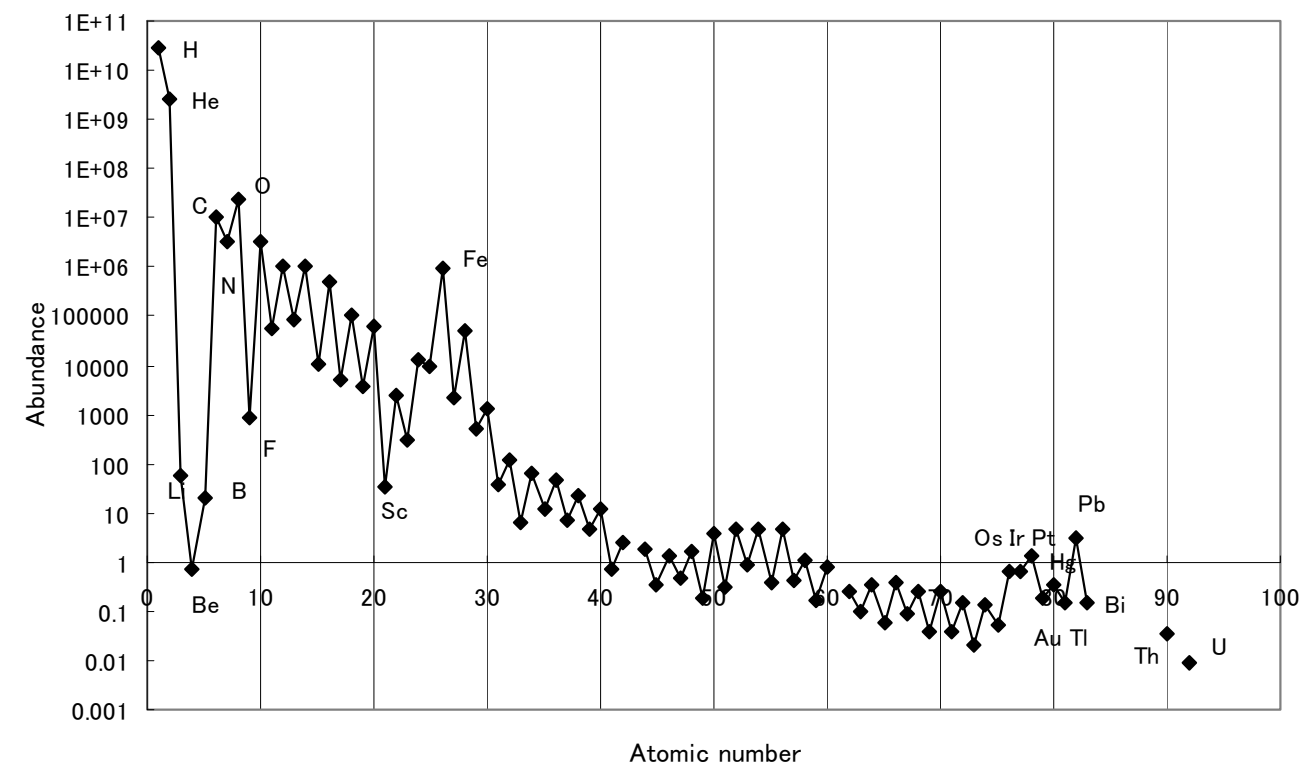

Figure 4. The Oddo-Harkins plot for the abundance of elements in the universe

Figure 5 shows the change of the reciprocal neutron multiple number with increasing atomic number. 


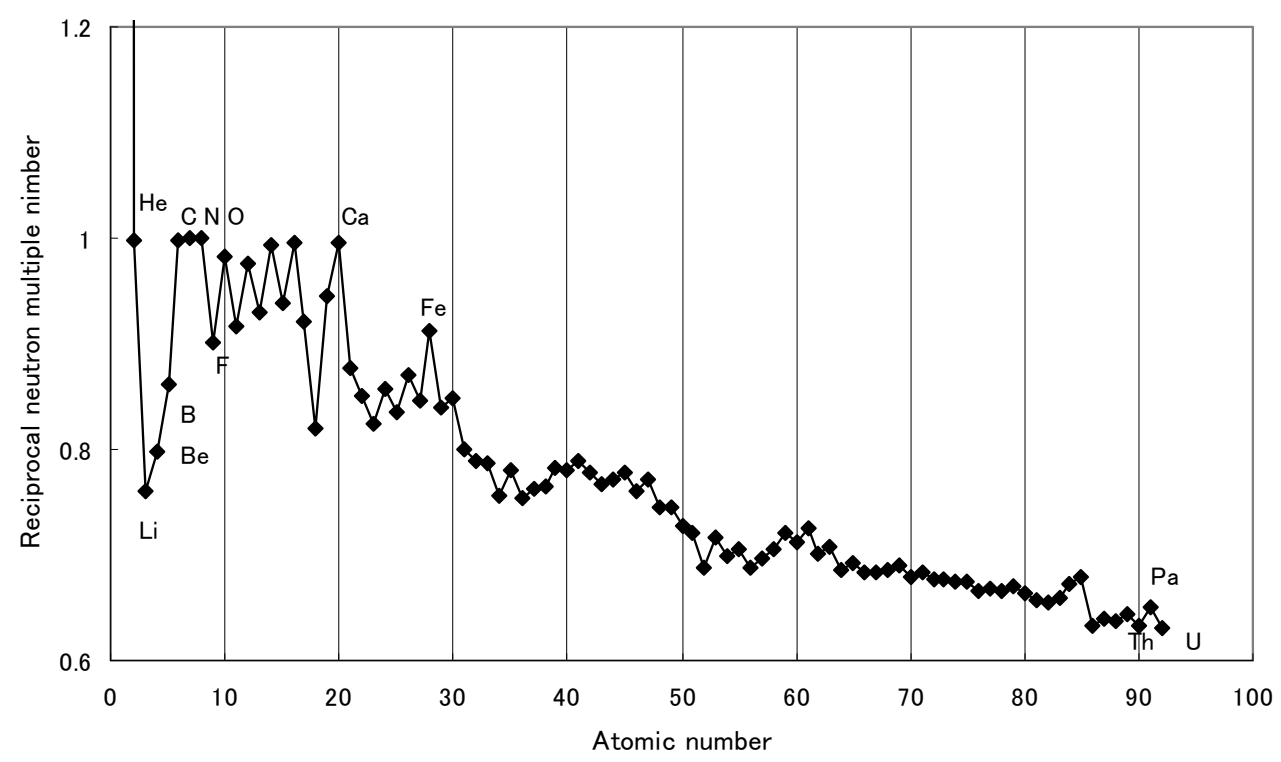

Figure 5. Reciprocal neutron multiple number with atomic number.

The abundance is inverse proportional to the neutron multiple number, therefore, the reciprocal neutron multiple number is convenient in some cases. With increasing atomic number, the reciprocal neutron multiple number decreases. But the elements Li, Be and B are exceptions in both Figure 4 and 5. The elements Li, Be and B needs more neutrons per proton to stabilize the nucleus than adjacent other elements. But these elements are not exceptions in Figure 3. They show the same trend as the other elements in the abundance vs. neutron multiple number plot shown in Figure 3.

It can be considered that, with increasing atomic number, the nucleus needs more neutrons per proton to shield the positive charges of protons and stabilize the nucleus. As a result, the frequency of atom formation decreases with increasing atomic number. In the elements with few protons such as $\mathrm{Li}, \mathrm{Be}$ and $\mathrm{B}$, they need many neutrons comparing to their few protons. One more neutron increases the neutron multiple number greatly at the elements with few protons. This is the reason why their abundance is small in spite that their atomic numbers are small. For instance, Be is synthesized from 2 Helium atoms, but Be with 8 mass number (The neutron multiple number is 1.) is unstable, and decomposes into He again (Kyougoku, 2011). Be is stable for the first time when it gets 5 neutrons, as is observed practically. Then, the neutron multiple number becomes 1.25 and the abundance decreases greatly according to the correlation between the abundance and the neutron multiple number shown in Figure 3 . Consequently, $\mathrm{Be}$ is located at the end of the minor trend in the lower part of the correlation. In addition, $\mathrm{Li}$ is located just on the lower part of the correlation line. B is located a little off the lower part of the correlation line, and is adjacent to Sc. They are not isolated in Figure 3, while they are exceptions in the Oddo-Harkins plot in Figure 4.

\section{Discussion}

The abundance of elements in the universe shows a good correlation with the neutron multiple numbers. With increasing neutron multiple number, the abundance in the logarithmic scale decreases linearly. It is understandable intuitionally that the elements which need more neutrons per proton are more difficult to be created. The Oddo-Harkins plot shown in Figure 4 is famous in the abundance of elements (Cherdyntsev, 1961). The abundance of elements decreases with increasing atomic number (Takigawa, 2013), but some exceptions are observed. The elements Li, Be and B show too small abundance and some reasons have been commented on it (Pagel, 2009; Aller, 1961). But they follow the general trend as is shown in Figure 3, where the abundance of elements is plotted versus neutron multiple number. $\mathrm{Li}, \mathrm{Be}$ and $\mathrm{B}$ are included in other elements and are no more exceptional. The exceptions of $\mathrm{Li}, \mathrm{Be}$ and $\mathrm{B}$ in the Oddo-Harkins plot are solved in Figure 3. It has been reported that the elements were created by various processes according to the atomic number (Aoki, 2004; Takigawa, 2013; Aller, 1961). But, totally all elements are ruled by the neutron multiple number.

Figure 5 shows the reciprocal neutron multiple number vs. atomic number plot of elements. This shows almost the same trend as the Oddo-Harkins plot. The Oddo-Harkins plot shows that the elements with even atomic numbers show larger abundance than the adjacent elements with odd atomic numbers. Figure 5 shows the same trend that 
the elements with even atomic numbers show larger reciprocal neutron multiple number than the adjacent elements with odd atomic numbers. It means that the elements with even atomic numbers are more stable, therefore they require less neutrons than those with odd atomic numbers. The elements which require less neutrons can be more easily synthesized and can be more common.

Figure 6 shows the relationship between the abundance and the neutron multiple number at the elements with odd atomic numbers and those with even atomic number, respectively.

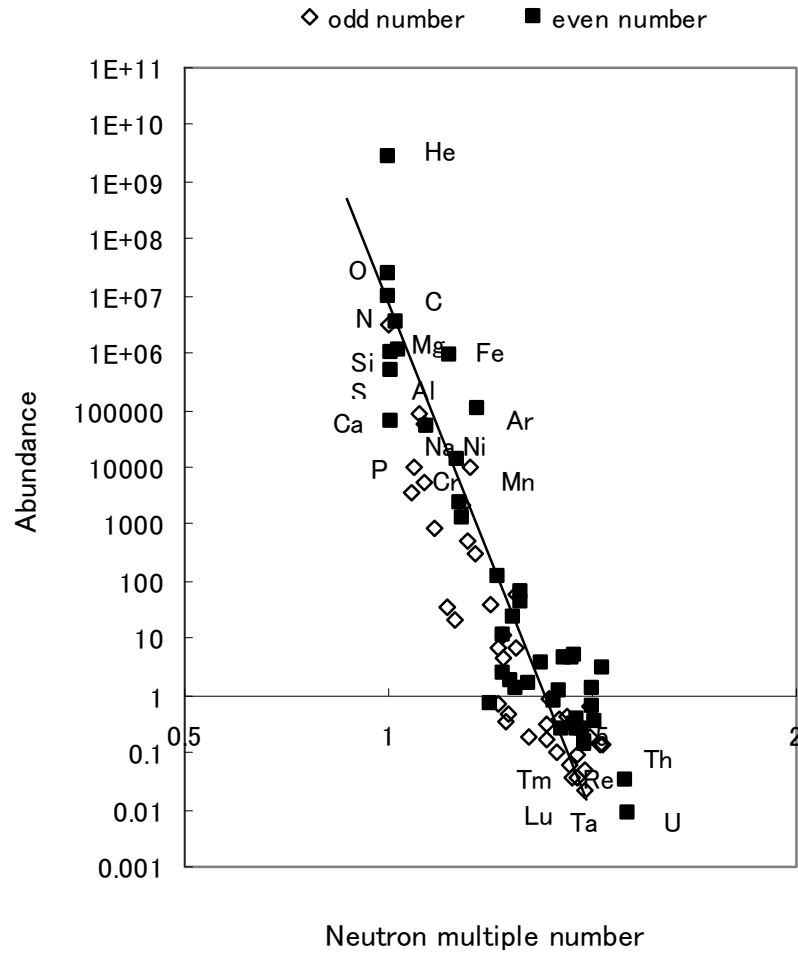

Figure 6. Correlation between the neutron multiple number and the abundance at both the elements of odd atomic numbers and those of even atomic numbers

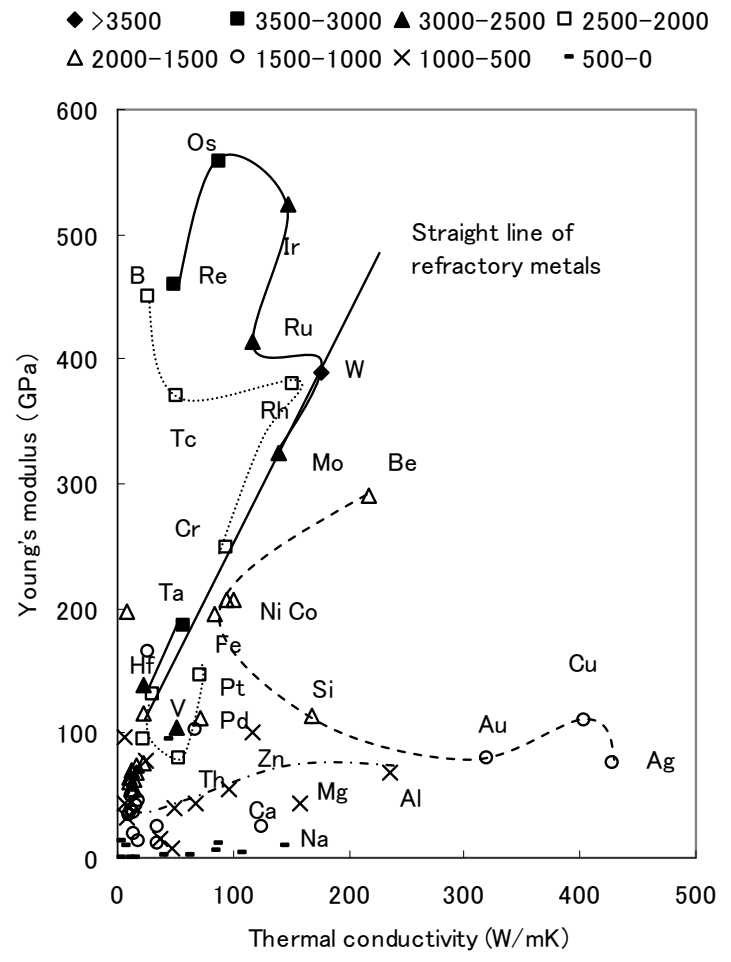

Figure 7. Distribution of the melting temperature of elements on the diagram

The elements with even atomic numbers dominate in the high abundance and low neutron multiple number region. The abundance of elements is not ruled by the atomic number, as the Oddo-Harkins plot says, but is ruled by the neutron multiple number.

Next, we study the effects of the neutron multiple number on the physical properties of elements.

Figure 2 shows that the neutron multiple number, that is, the structure of the nucleus is relevant to the physical properties of elements. The elements with small neutron multiple numbers show the self-compensating combination of Young's modulus and thermal conductivity.

Figure 7 shows the distribution of the melting temperature of elements on the TC-YM diagram (Mae, 2016).

The figures in the legend show the range of the melting temperature in Kelvin. In the melting temperature, the elements of high Young's modulus, Re, Os, Ir, Ru, W, and Mo, show the highest values, and the trend decreases gradually along the straight line of refractory metals and reaches the elements of low Young's modulus Ta and Hf. In contrast, the elements of low melting temperatures are distributed in low Young's modulus region. This type of property will be called "the property of Young's modulus type", because the effect of Young's modulus is dominant.

Figure 8 shows a correlation between the melting temperature of elements and the neutron multiple number.

Figure 8 shows only the results regarding to the elements of high Young' moduli over $200 \mathrm{GPa}$. Some trend can be seen that with increasing neutron multiple number, the melting temperature increases. The elements with low Young's moduli do not show the trend. 


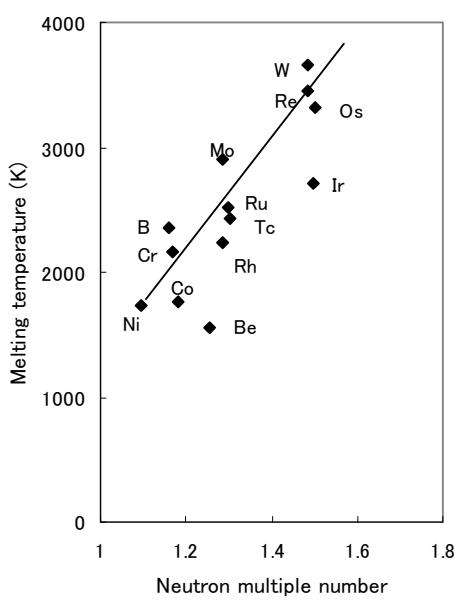

Figure 8. Correlation between the melting temperature and the neutron multiple number at the elements with the

Young's moduli above $200 \mathrm{GPa}$

\section{Conclusions}

The abundance of elements shows a unique pattern drawing a quadrant on the TC-YM diagram. Similarly, the neutron multiple number, defined as the number of neutron per proton in the nucleus, shows the same pattern. A good correlation that, with increasing neutron multiple number, the abundance of elements decreases, is recognized. The exceptional elements in the Oddo-Harkins plot $\mathrm{Li}, \mathrm{Be}$ and $\mathrm{B}$ are included in that correlation. The abundance of elements is not ruled by the atomic number, as the Oddo-Harkins plot says, but is ruled by the neutron multiple number. The elements with small neutron multiple number are more easily synthesized than the elements with large neutron multiple number.

Moreover, the atoms with even proton numbers need less neutrons than those with odd proton numbers, and become to have larger abundance.

Furthermore, the neutron multiple number affects the material properties of elements. The elements with small neutron multiple numbers have the self-compensating combination of Young's modulus and thermal conductivity. The effect of the neutron multiple number on the material properties of elements can be seen also in the melting temperatures of high Young's modulus elements.

\section{References}

Aller, L. H. (1961). The abundance of the element. New York, NY: Interscience Publishing.

Aoki, W. (2004). Cosmological history of materials. Tokyo: Shin-nihon Shuppan.

Cherdyntsev, V. V. (1961). Abundance of chemical elements. Chicago: University of Chicago.

Kumano, S. (2015). Nuclear physics. Tokyo: Kyouritu-shuppan.

Kyougoku, K. (2011). Structure of the universe and elementary particles. Tokyo: Ascii Media Works.

Mae, Y. (2016). What the Draken-Gurry plot means about the solubility of elements in metals. Metall. Mater. Trans. A, 47, 6498-6506. https://doi.org/10.1007/s11661-016-3730-1

Mae, Y. (2017). Anthropic principle observed in the material properties of Fe. J. Mater. Sci. Res., 6, 11-19. https://doi.org/10.5529/jmsr.v6n3p11

Oda, M. (1987). Big cosmological dictionary. Tokyo: Maruzen.

Ono, M. (2008). Understanding elements. Tokyo: Gijutsu-Hyouron-sha.

Pagel, B. (2009). Nucleosynthesis and chemical evolution of galaxies. Cambridge: Cambridge University Press.

Takigawa, N. (2013). Nuclear physics. Tokyo: Asakura Book-store.

\section{Copyrights}

Copyright for this article is retained by the author(s), with first publication rights granted to the journal.

This is an open-access article distributed under the terms and conditions of the Creative Commons Attribution license (http://creativecommons.org/licenses/by/4.0/). 